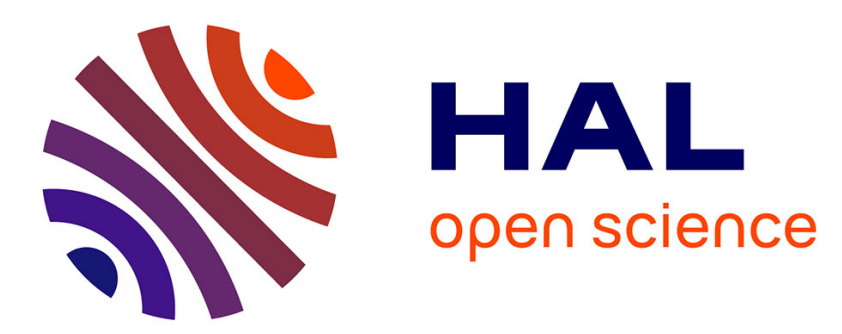

\title{
Style of practice and assortative mating: a recursive probit analysis of cesarean section scheduling in Italy
}

Daniele Fabbri, Chiara Monfardini

\section{To cite this version:}

Daniele Fabbri, Chiara Monfardini. Style of practice and assortative mating: a recursive probit analysis of cesarean section scheduling in Italy. Applied Economics, 2008, 40 (11), pp.1411-1423. 10.1080/00036840600771395. hal-00582096

\section{HAL Id: hal-00582096 https://hal.science/hal-00582096}

Submitted on 1 Apr 2011

HAL is a multi-disciplinary open access archive for the deposit and dissemination of scientific research documents, whether they are published or not. The documents may come from teaching and research institutions in France or abroad, or from public or private research centers.
L'archive ouverte pluridisciplinaire HAL, est destinée au dépôt et à la diffusion de documents scientifiques de niveau recherche, publiés ou non, émanant des établissements d'enseignement et de recherche français ou étrangers, des laboratoires publics ou privés. 


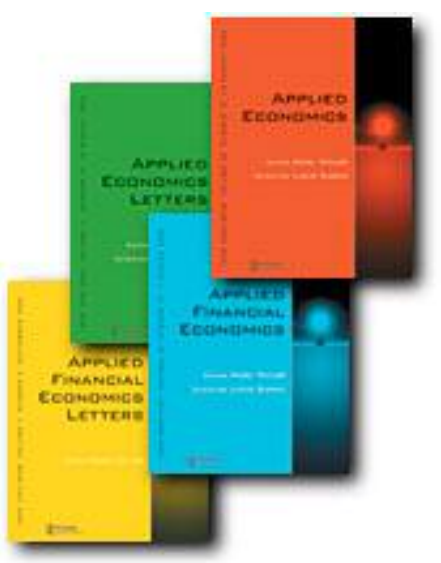

Style of practice and assortative mating: a recursive probit analysis of cesarean section scheduling in Italy

\begin{tabular}{|r|l|}
\hline Journal: & Applied Economics \\
\hline Manuscript ID: & APE-06-0175.R1 \\
\hline Journal Selection: & Applied Economics \\
\hline JEL Code: & $\begin{array}{l}\text { C35 - Discrete Regression and Qualitative Choice Models < C3 - } \\
\text { Econometric Methods: Multiple/Simultaneous Equation Models < C - } \\
\text { Mathematical and Quantitative Methods, I11 - Analysis of Health } \\
\text { Care Markets < I1 - Health < I - Health, Education, and Welfare, } \\
\text { C52 - Model Evaluation and Testing < C5 - Econometric Modeling < } \\
\text { C - Mathematical and Quantitative Methods }\end{array}$ \\
\hline Keywords: & $\begin{array}{l}\text { practice variation, assortative mating, cesarean section scheduling, } \\
\text { recursive probit model }\end{array}$ \\
\hline
\end{tabular}




\title{
Style of practice and assortative mating:
}

\author{
a recursive probit analysis of cesarean section scheduling in Italy
}

\author{
Daniele Fabbri*, Chiara Monfardini
}

Department of Economics, University of Bologna,

Piazza Scaravilli 2, 40126 Bologna, Italy.

\begin{abstract}
We study practice variation in scheduling of cesarean section delivery across public and private hospitals in Italy. Adopting a novel perspective, we look at the role played by patients' preferences for the treatment. The recursive probit model is revisited as a useful tool to assess the presence of assortative mating of patients and provider driven by style of practice. According to our evidence the propensity to scheduling a cesarean section is codetermined with patient self-sorting into hospital types. We measure a significantly higher inclination to practice cesarean section scheduling in private hospitals and conclude that assortative mating is of minor relevance in our case, even if we cannot exclude it to be present.
\end{abstract}

Keywords practice variation, assortative mating, cesarean section scheduling, recursive probit model

J.E.L. I11, C35, C52

Running title: Style of practice and assortative mating

*corresponding author: Department of Economics, University of Bologna, Piazza Scaravilli, 2 - 40126 BOLOGNA. Italy. e-mail: dfabbri@economia.unibo.it. 


\section{Introduction}

Persistent variation across geographic areas and across providers in the use of medical procedures represents a largely unexplained basic evidence in the health economics literature. A common view is that such pattern of variation emerges out of an asymmetric relationtioship between a subject patient and a dominant physician basically because of the disagreement across physician groups about the shape of the health production function, i.e. the function transforming medical care into health outcomes. This presumption seems hard to be rejected in the case of pure regional variation (Wennberg and Gittelsohn, 1973). When we come to physician practice at least part of observed variation can be plausibly ascribed to a process of "assortative mating of doctors who are aggressive with patients who prefer aggressive treatment" (Phelps, 2000, page 251).

The relation between style of practice and assortative mating has been surprisingly neglected in the literature despite its strong implications for patients' welfare. Practice variation leads to a welfare loss whenever patients share homogenous preferences for "product" attributes, i.e. there is a single treatment that well informed patients prefer (Ryan and Hughes, 1997, San Miguel et al., 2000). Phelps and Mooney (1993) suggest that such a loss is of a comparable magnitude to the one emerging out of ex-post moral hazard in health insurance contracts and likewise impractical to be entirely recaptured to society. Targeted policies are invoked on a benefit-cost basis in the purpose of reducing welfare losses until marginal benefits pair marginal costs. Huge investments in the production and dissemination of novel evidence about the efficacy of various medical procedures are quite easy to justify in this framework (Phelps, 2000). On the contrary, whenever consumers have heterogenous preferences, provided they are able to identify provider treatment styles and are free to choose accordingly, then "product variety" will improve welfare. Ascertaining the existence of assortative mating mechanisms therefore lessens the argument in favor of active policies aimed at reducing practice variation and at the same time provides a rationale for policies aimed at improving patients' awareness of providers style of practice.

In this paper we take a first step in this direction and bring into focus the main ingredients to evaluate the relevance of assortative mating in healthcare markets. Generally speaking, the analyzed 
case should be characterized by difference in style of practice across providers, patients' ability to observe provider's style of practice and quality, patients' heterogenous preferences for alternative treatments and free choice among alternative providers. Accordingly, the empirical model must be able to identify sistematic variation in practice across providers net of a full set of patients covariates, and to account for nonrandom selection of patients into hospitals. The source of the latter mechanism can be twofold: patients unobserved frailty and patients unobserved preferences for a given treatment. We show in the paper under which circumstances it is possible to interpret this self-selection mechanism as evidence of assortative mating.

We deal on a case study which has attracted a massive attention in the health economics literature. Cesarean section (CS) is one of the most common surgical procedure worldwide. In Italy and the US it is the second most frequent procedure with respectively 200,000 and 900,000 CS performed yearly. Quite some concern has been expressed about the increasing adoption of such a technology for birth beyond the realm of clinical abuse. According to OECD data CS incidence rose in developed countries from $6 \%$ in 1970 to more than $20 \%$ in 1998 . This evidence conflicts with WHO (1985) recommendations on appropriate technology for birth, suggesting that "there is no justification, in any specific geographic region, to have more than 10-15\% cesarean section births". Similar clinical guidelines have been proposed by the Agency for Healthcare Research and Quality (2002) and by the US Department of Health and Human Resources (2000). The conflict between clinical evidence and suggested guidelines stimulated quite some research efforts in the health economics literature to gain insights about the reasons behind this apparent overuse. Economists' contributions are deeply rooted into the so called Physician Demand Induction framework, i.e. the idea that in the face of negative income shocks, physicians may exploit their agency relationship with patients by providing excessive care (McGuire and Pauly, 1991). Income shocks exploited in the literature arise from competitive pressure in the local market as measured by variation in physician density (Cromwell and Mitchell, 1986), from exogenous reduction in reimbursement tariffs (Gruber et al., 1999), from declining fertility (Gruber and Owings, 1996), from increasing threat of malpractice suit (Dubay et al., 1999). The role played 
by patients' preferences has been left unexplored in the health economics literature despite anecdoctal evidence of its relevance. ${ }^{1}$ A currently prevailing wisdom in the health policy literature seems to favour the idea that obstetricians' and patients' preferences jointly play a major role in determing delivery procedures (Paterson-Brown, 1998, Minkoff and Chevernak, 2003).

We present in this paper novel evidence about variation in treatment style for deliveries across two classes of providers, public and private hospitals, on a nationwide representative sample of Italian women in childbirth. We measure treatment style as the proportion of deliveries performed by CS in the two classes of hospitals. As it comes clear by looking at Table 1 CS rates are markedly different across the two classes mainly because of the private hospitals inclination to schedule CS. Conditional on laboring CS rates are indeed quite similar. Our general conjecture is that this difference in style of practice can be recognized by patients and drive, at least partially, a nonrandom self-sorting of patients into the two hospitals' types. Actually, scheduled CS cannot be viewed as a purely unilateral clinical decision a physician makes on behalf of his patient. It is made in large advance, allowing the patient to switch to another provider in case she disagrees with the scheduled decision. Moreover the extent of information asymmetry involved here between the physician and his patient seems quite limited: the set of alternative technologies for birth is small and the social knowledge about each alternative is spread and diffuse also in terms of their clinical implications. Finally patients preferences for the treatment are influenced by idiosyncratic factors like aversion to risk for the newborn, aversion to pain and suffering, taste for natural processes. These general features make scheduled CS a favorable case study for ascertaining the existence of assortative mating mechanisms. Some further aspects peculiar to our Italian case study are worth noticing here. First of all, in the italian NHS women are completely free to choose the treating hospital -public or private- with no out-of-pocket payments. Secondly, public

\footnotetext{
${ }^{1}$ According to MacKenzie (1999) in $199630 \%$ of total antepartum cesarean section performed at the John Radcliffe Hospital in Oxford are on maternal request. This phenomenon was almost absent in the previous two decades. Al-Mufti et al. (1997) suggest that $31 \%$ of London female obstetricians with an uncomplicated singleton pregnancy at term would choose an elective CS for themselves. Lo (2003) provides evidence of significant increase in CS due to preferences for specific birthdays in China.
} 
and private hospitals are naturally sorted in terms of quality and infra-structural capacity. Public hospitals have emergency surgical capacity and newborn intensive care units (WHO, 1985, recommends that "natural deliveries after a caesarean should normally be encouraged wherever emergency surgical capacity is available"). On the other hand, private hospitals do not have emergency room and therefore are not allowed to admit on an emergency. Finally the presence of teaching personnel increases the role of professional and deontic rewards in the public leading to a higher propensity to improve clinical practices and to adopt the more appropriate ones. Because of these reasons, public hospitals are nationwide perceived in Italy as of higher quality for delivery. We exploit this quality difference in the interpretation of women self-selection mechanism into hospital type.

\section{INSERT TABLE 1 HERE}

To motivate our empirical analysis of assortative mating in scheduled CS we develop an interpretative model for the hospital choice and the delivery mode that incorporates the role of patient preferences for clinical and non clinical quality, aversion to risk and pain. We consider the scheduling decision as the possible outcome of a bargaining process between the physician and his patient. This process is conditioned, on the physician side, by deontic reasons and adherence to professional norms, financial incentives, overall clinical endowments in the operating hospital, fear for malpractice suit. On the patient side, bargaining is affected by preferences for the treatment, preferences for clinical and non clinical quality.

The econometric model we adopt acknowledges the binary nature of the endogenous variable represented by treatment: planned CS versus attempt of natural delivery (ND). The analysis of practice variation across public and private providers is performed by including among the determinants for the probability of the treatment a dummy indicating the provider chosen by the patient, beside a set of observable risk factors. Scheduling is jointly decided with provider choice, through an individual process in which patients' preferences for the alternative treatments and information on provider's style of practice play a major role. This brings about self-selection of patients into providers based on observables and unobservables characteristics that also determine the given treatment, making the provider 
dummy variable potentially endogenous. An adequate model to represent this phenomenon is the recursive probit model with endogenous dummy (Heckman, 1978). The main objects of the inference are the coefficient of the potentially endogenous dummy variable indicating the chosen provider, and the correlation coefficient between the error terms of the two equations. Through the first coefficient it is possible to evaluate the existence and the extent of the difference in style of practice across providers. The second coefficient signals the presence of a self-selection mechanism operating through unobservable variables. We explain in the paper that in presence of assortative mating both coefficients are expected to be non null. We find that the propensity to scheduling a CS across providers is codetermined with patient self-sorting into hospital type as hinted by the battery of exogenity tests we apply. We measure a significantly higher inclination to practice CS scheduling in private hospitals and conclude that assortative mating in the public-private dimension is of minor relevance, even if we cannot exclude it to be present.

The paper proceeds as follows. In section 2 we elaborate an interpretative model for the hospital choice and the delivery mode. Section 3 presents our empirical model. Section 4 illustrates our case study, presents the estimation results and their interpretation. Section 6 contains some final remarks.

\section{Understanding the decision process for cesarean section schedul- ing and hospital choice}

We outline here an interpretative model for hospital choice and delivery mode accounting for some peculiar features in our case study. In particular we emphasise the role played by patients' preferences for the treatment.

We consider that each individual belonging to the population of women in childbirth is described by an indicator $r$ comprising all risk factors for a difficult delivery. The population is distributed between $\underline{r}$ and $\bar{r}$ in ascending order of risk. There are only two hospitals where a woman can deliver in: a private (PR-h) and a public one (PU-h). ${ }^{2}$

\footnotetext{
${ }^{2}$ For ease of exposition we will consider the obstetrician and the hospital where he operates as interchangeable. In a
} 
The PU-h obstetrician provides appropriate treatments as far as he "unilaterally" follows professional guidelines in order to gain deontic premiums. ${ }^{3}$ The rule is like the following: if the women is of type $r$ where $\bar{r} \geqq r \geqq r_{S_{P U}}>\underline{r}$ then schedule her a CS (action $S_{P U}$, where S stands for "scheduled" CS and the uppercase indicates that the clinical decision is appropriate); try a ND and therefore enter labor (action $L_{P U}$, where $\mathrm{L}$ stands for "labor") otherwise. No bargaining over the treatment is accomodated by the PU-h.

The PR-h obstetrician is prompt to accomodate patients' preferences in accordance to his own objectives and therefore to bargain with the patient under the threat of patient's switch to the PUh. He might propose to his patient an appropriate scheduled CS $\left(S_{P R}\right)$, a non appropriate scheduled CS $\left(s_{P R}\right)$, or to attempt a ND $\left(L_{P R}\right) . S_{P R}$ is given according to a more lenient decision rule with respect to the PU-h (i.e. $r_{S_{P R}}<r_{S_{P U}}$ ). This is due to staffing and technical equipment limitations, as generally argued by Minkoff and Chevernak (2003), in the PR-h. Therefore appropriate scheduled CS is equally frequent across the two hospital's types conditional on staffing and technical equipment. $s_{P R}$ is administred according to an even more lenient rule $\left(r_{s_{P R}}<r_{S_{P R}}<r_{S_{P U}}\right)$, i.e. a rule that leads to a more frequent scheduling of a CS even after controlling for differences in staffing and technical equipment.

Coming to the payoffs, as far as the PU-h is assumed to behave according to automatic unilateral rules, it is not an agent in our simple game. It simply represents the patient's outside option. Concerning the incentives for the PR-h, we pose that by performing a $S_{P R}$ the hospital/obstetrician gains an economic rent, $A$, comprising the anticipated diffential reimbursement of CS, time cost savings and lower efforts with respect to ND. By performing $s_{P R}$ the economic rent $A$ is reduced of a positive amount $a<A$ comprising the monetary equivalent for deontic penalties suffered by the obstetrician that overlooks his Hippocratic hoath. We assume that the deontic penalty is a decreasing function of the patient's risk indicator, $a(r)$, with $a_{r}<0 . L_{P R}$ is associated to a positive payoff $b$ reflecting the sense we assume that the hospital is under the complete control of the staffed physicians and therefore implied agency problems are totally absent.

${ }^{3}$ Frank (2004) discusses unilateralism in clinical decisions within the paradigm of behavioral economics. 
anticipated economic rents, plausibly smaller than those accruing for performing a scheduled CS, net of deontic penalties for performing a CS after labor. Therefore the payoff $b$ is definitely lower than $A-a(r)$. We are now able to characterize the cutoff value for $s_{P R}, r_{s_{P R}}$, as the value of women risk indicator such that $A-a\left(r_{s_{P R}}\right)=b$. The decision to enter labor is always appropriate as far as there is no relative convenience to its overuse: it is never administered to a high risk patient, i.e. with $r>r_{S_{P R}}$.

We finally describe patient's payoffs. Let $B_{H}^{c}$ denote the payoff accruing to the patient in case her chosen hospital $H$ (with $H=$ PU-h, PR-h) adopts the clinical decision $c$, where $c \in C=(S, s, L)$. Consider first the riskiest patient, i.e. that having a risk indicator $r>r_{S_{P U}}$. She will value the highest the opportunity to receive a scheduled CS in PU-h. The very high risk patient alwas refers to PU-h as far as, conditional on her risk factors, she receives there an appropriate scheduled CS. Referring to PU-h is her best choice given that higher risk patients demand good unilateral clinical decisions. Once we exclude the riskiest patients then the structure of patient's payoff have implications on the joint decisions of CS scheduling and hospital choice.

When the patient have a strong aversion to a painful and risky ND even in the public hospital, i.e. $B_{P R}^{s}>B_{H}^{L}$, she will opt for the PR-h. We call this the "preference for scheduled CS" case. It is worth noticing that in this case if $r \in\left[r_{s_{P R}}, r_{s_{P U}}\right)$ the patient opt for the PR-h and accept the unilateral clinical decision adopted there, i.e. to schedule an inappropriate CS. On the contrary when we turn to the very low risk tail, i.e. patient with $r<r_{s_{P R}}$, PR-h obstetrician is to make her entering labor: the payoff for an appropriate ND is higher than that accruing to him in case of an inappropriate scheduled CS because of large deontic penalty. In this case, a bargaining between the patient and the PR-h might emerge. Her threat of switch to the PU-h makes the bargaining over scheduled CS beneficial for the PR-h in face of the loss of a patient. Gain from bargaining is equal to $A-a(r)$; concomitantly, for the patient it is equal to $B_{P R}^{s}-B_{P U}^{L}$. Assuming a very simple Nash bargaining framework ${ }^{4}$ we can state that a patient with risk profile $r<r_{s_{P R}}$ will refer to PR-h and receive an inappropriate scheduled CS as far as the the following inequality is satisfied:

\footnotetext{
${ }^{4}$ A similar framework has been adopted for dealing with the hospital-insurer relationship by Brooks et al. (1997) and Maude-Griffin et al. (2004).
} 


$$
(A-a(r))^{\gamma} \cdot\left(B_{P R}^{s}-B_{P U}^{L}\right)^{1-\gamma}>b^{\gamma} \cdot\left(B_{P R}^{L}-B_{P U}^{L}\right)^{1-\gamma}
$$

where $\gamma \in[0,1]$ represents obstetrician's bargaining power and $1-\gamma$ that of his patient. The choice of a PR-h and the concomitant scheduling of an inappropriate CS is therefore more (less) frequent the lower (higher) is the bargaining power of the hospital/obstetrician, the stronger (weaker) are economic incentives on the PR-h, the higher (lower) is patient's riskiness, the larger (lower) is patient aversion to pain and suffering.

Another relevant preference structure is the one that describes a patient highly valuing the (non clinical) quality aspects provided by a private hospital. Within this preference structure a case that is worth discussing is the "preference for a ND in a pleasant environment", while the one concerned with scheduled CS is similar to the "preference for scheduled CS" we discussed above. In this case, i.e. when $B_{P R}^{L}>B_{P R}^{S}>B_{P R}^{s}>B_{P U}^{L}$, a patient in the middle of the risk distribution, $r \in\left(r_{s_{P R}}, r_{S_{P U}}\right)$, might force the PR-h to bargain over the choice of not scheduling a CS. ${ }^{5}$ However, despite possible, such a bargaining is ineffective as far as once the patient enters labor the clinical decision becomes purely unilateral and therefore is not contractible ex-ante.

\section{The empirical framework}

We depict here a simple empirical framework to assess the existence of assortative mating. Coherently with our interpretative model we start by defining a latent variable indicator $s_{i}^{*}=f\left(r_{i}\right)$ so that the

\footnotetext{
${ }^{5}$ The decision not to schedule a CS (either appropriate or inappropriate) emerges provided the following inequalities

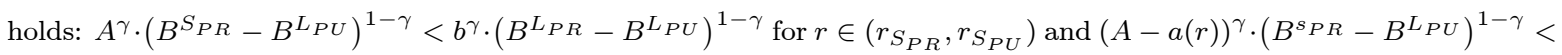
$b^{\gamma} \cdot\left(B^{L_{P R}}-B^{L_{P U}}\right)^{1-\gamma}$ for $r \in\left(r_{s_{P R}}, r_{S_{P R}}\right)$. The latter inequality, referring to the less risky patients in the middle of the distribution, is more easily met. The economic rent accruing to the provider net of deontic penalties for inappropriate planned CS is smaller and patient gain over an admission in the PU-h is smaller as well in case an inappropriate CS is proposed. Notice that no bargaining arises if $r<r_{s_{P R}}$ given that both agents agree on the decision to attempt to a ND.
} 
dichotomus choice of scheduled CS vs the attempt of a ND $s_{i}$ is observed according to the rule:

$$
\left\{\begin{array}{lll}
\text { "schedule a CS": } & s_{i}=1 \text { if } \quad s_{i}^{*}>0 \\
\text { "attempt a ND": } & s_{i}=0 \text { if } \quad s_{i}^{*} \leq 0
\end{array}\right.
$$

Such a choice can be interpreted, conditionally upon risk and predisposing factors $r_{i}$, as an "unilateral", purely deontic decision rule for a patient delivering in a public hospital. The decision rule shifts from such a "golden standard" in case the woman chooses to deliver in a private hospital. In a sense we consider the obstetricians operating in public hospitals as "professional leaders" setting the professional norm the collegues operating in private hospitals look at. Assuming a parametric linear specification, the scheduling decision emerges then according to the latent regression:

$$
s_{i}^{*}=\delta_{1} \operatorname{priv}_{i}+f\left(r_{i}\right)=\delta_{1} \operatorname{priv}_{i}+\delta_{2} z_{i}+u_{s i}
$$

where $\operatorname{priv}_{i}$ is a dichotomous variable indicating delivery in private hospital, $z_{i}$ is a vector collecting exogenous observable risk and predisposing factors, while $u_{s i}$ is a stochastic term capturing all the unmeasured characteristics of the woman. The above equation reflects the outcome of a joint decision process involving the two agents. We would like to interpret the difference in probability of scheduling in private hospitals with respect to public as a measure of private departure from the public appropriate, professional norm.

However, as we argued above, the hospital choice is concomitant to the scheduling process, in some cases even subject to strategic bargaining considerations. The woman may choose to opt out of a public hospital admission aware of her health conditions, hospital characteristics and the clinical decision rule adopted there. Therefore the two classes of hospital will attract women with different preferences and different clinical characteristics. Some of these determinants are observed, other are not, forcing us to consider equation 1 jointly with a hospital choice process. This process is driven by the following stochastic latent indicator:

$$
\operatorname{priv}_{i}^{*}=\beta_{1}^{\prime} x_{i}+u_{h i}
$$


and determines the observable variable priv $_{i}$ according to the rule:

$$
\left\{\begin{array}{l}
\text { "refer to PR-h" } \text { priv }_{i}=1 \quad \text { if } \quad \text { priv }_{i}^{*}>0 \\
\text { "refer to PU-h" } \quad \text { priv }_{i}=0 \quad \text { if } \quad \text { priv }_{i}^{*} \leq 0
\end{array}\right.
$$

The vector $x_{i}$ contains exogenous observable risk factors and socio-economic characteristics of the woman and $u_{h i}$ is a stochastic error term. Omission of common unobservable variables in equations 1 and 2 introduces a correlation pattern between the two stochastic components $\left(u_{s i}, u_{h i}\right)$. Adding to equations 1 and 2 the assumption that the latter are independently and identically distributed as bivariate normal:

$$
\left(\begin{array}{l}
u_{s i} \\
u_{h i}
\end{array}\right) \sim I I D N\left(\left[\begin{array}{l}
0 \\
0
\end{array}\right],\left[\begin{array}{ll}
1 & \rho \\
\rho & 1
\end{array}\right]\right)
$$

results in a bivariate probit model with endogenous dummy. This model belongs to the general class of simultaneous equation models with both continuous and discrete endogenous variables introduced by Heckman (1978). Maddala (1983) lists this (as Model 6) among the recursive models for dichotomous choice. The recursive structure builds on a first reduced form equation for the potentially endogenous dummy (the hospital type choice equation 2 in our case)- and a second structural form equation determining the outcome of interest (the scheduling decision process 1). ${ }^{6}$

Some hints on the interpretation of the correlation coefficient $\rho$ in our modelling exercises can be obtained putting forward the following simplifying decomposition of the two error terms of the model:

$$
\begin{aligned}
& u_{s i}=\varphi_{1} \varepsilon_{r i}+\varphi_{2} \varepsilon_{p i}+\eta_{1 i} \\
& u_{h i}=\gamma_{1} \varepsilon_{r i}+\gamma_{2} \varepsilon_{p i}+\eta_{2 i}
\end{aligned}
$$

where $\varepsilon_{r i}$ indicates unobserved adverse clinical conditions relevant for delivery, $\varepsilon_{p i}$ represents her unobservable tastes in favour of a CS (like degree of aversion to pain and suffering, taste for natural

\footnotetext{
${ }^{6}$ In health economics the model has been used to analyse the effect of supplemental insurance ownership on dycotomous health demand indicators (see Holly et al., 1998, Buchmueller et al., 2004) and to explore endogeneity of self-reported disability measure for the decision to apply for social benefits (Benitez-Silva et al., 2004).
} 
processes), while $\eta_{1 i}$ and $\eta_{2 i}$ are the residual unobserved random component of the two latent indicators, normally distributed with zero mean, variances $\sigma_{\eta 1}^{2}$ and $\sigma_{\eta 2}^{2}$ respectively, uncorrelated with each other. We assume that $\varepsilon_{r i}$ and $\varepsilon_{p i}$ are normal, zero mean, uncorrelated with each other and with $\eta_{1 i}$ and $\eta_{2 i}$, with variances $\sigma_{r}^{2}$ and $\sigma_{p}^{2}$ respectively. ${ }^{7}$ In this setting, the correlation between the error terms of the two probit equations arises only from the two common unobserved components $\varepsilon_{r i}$ and $\varepsilon_{p i}: \rho=E\left(u_{1 i}, u_{2 i}\right)=\gamma_{1} \varphi_{1} \sigma_{r}^{2}+\gamma_{2} \varphi_{2} \sigma_{p}^{2}=\rho_{r}+\rho_{p}$. This splits the correlation coefficient into two parts: the first term $\rho_{r}$ captures a selection mechanism related to clinical risk, the second one $\rho_{p}$, relates to the preferences of the woman. The coefficients $\gamma_{1}, \gamma_{2}, \varphi_{1}, \varphi_{2}$ are clearly not identifiable, but are inserted because speculating on their sign according to the assumptions presented in section 2 we are able to provide some possible interpretations of the identified correlation coefficient $\rho$. Coherently with the discussion of the previous section we can derive the following implications. Concerning the risk component, $\gamma_{1}<0$ and $\varphi_{1}>0$, i.e. $\rho_{r}<0$. This means that the more frail patient refer to the higher quality hospital, the public one in our case. This kind of nonrandom selection to hospitals has been strongly evidenced by Geweke et al. (2003). Turning to the unobservable preference component, our definition of $\varepsilon_{p i}$ implies $\varphi_{2}>0$, while the sign of $\gamma_{2}$ is more controversial. Recalling the alternative preference patterns sketched above, in the "preference for scheduled CS" case we expect $\gamma_{2}>0$, while the "preference for ND in a pleasant environment" case is compatible with $\gamma_{2}<0$. Following our theoretical framework, we discard this last pattern as unplausible. Therefore, it is possible to state that the preference component $\rho_{p}$ is positive. When the self-sorting mechanism due to unobservable preferences can be ascribed to a recognizable practice variation across providers, then assortative mating can be claimed to be in place. In the above context, this implies $\rho_{p}>0$ and $\delta_{1}>0$. Given that the identified parameter is $\rho$, the practical implementation of a test for the presence of assortative mating is confronted with the difficulty represented by the presence of the risk component $\rho_{r}$. The negative (positive) sign of $\rho$ testifies that the risk component $\rho_{r}$ (preference component $\rho_{p}$ ) prevails upon the other. The relative importance of the two components is an empirical matter. The richer the set of risk control

${ }^{7}$ The variances of the two idiosyncratic components, $\sigma_{\eta 1}^{2}$ and $\sigma_{\eta 2}^{2}$ are assumed to get values making the normalization $\operatorname{Var}\left(u_{1 i}\right)=\operatorname{Var}\left(u_{2 i}\right)=1$ to hold. 
available to the researcher the larger will be the role played by the patient unobserved preference and the scope for assessing the existence of assortative mating.

The implications for the empirical tests are the following. First, when the correlation coefficient is found to be statistically equal to zero, the evidence about assortative mating is inconclusive, but the resulting exogeneity of the dummy allows to use only the treatment equation for investigating practice variation. Second, a significant impact of provider's dummy toghether with a positive correlation coefficient testifies the existence of assortative mating (while a negative correlation coefficient does not allow to draw any conclusion on this mechanism).

\section{Scheduling Cesarean Section delivery and self-selection into hospital types in Italy}

\subsection{Data description}

We work on a dataset coming from the "Indagine Statistica Multiscopo sulle Famiglie: condizioni di salute e ricorso ai servizi sanitari" (ISMF), a national household survey conducted by the Italian National Institute of Statistics (ISTAT) every 5 years. The last available survey was conducted from september 1999 to august 2000 when a sample of 40119 households were interviewed. The survey provides a full account of individual health condition, health care utilization, biometric parameters plus socio-economic status (education, working condition) and other relevant economic variables like complementary private health insurance holding. In this study we exploit a section of the survey focussing on the last delivery experienced by female components of each sampled household in the five years before the interview. Delivery experience is described in an individual self-compiled part of the survey. Data about mode of delivery, health problems suffered and therapies underwent during pregnancy and delivery are self-reported. Therefore we do not rely on approximate methods based on administrative data, like the one used by Epstein and Nicholson (2005), to identify CS scheduling. This is critical in case of strategical miscoding. We have 4516 women filling in this section of the survey for 
a corresponding number of deliveries occurring in the four years before the interview.

We control for a full set of regressors (see the following Table 2 for a list, and table A.1 in the Appendix for descriptive statistics) including individual predisposing risk factors for CS delivery and some socioeconomic variables. Within our interpretative framework, we expect risk factor variables to affect negatively the probability of referring to a private hospital and positively the CS scheduling probability. Turning to the socioeconomic regressors, education captures heterogeneity in preferences in both equations and the direction of its effect is more controversial. It is important to clarify that as far as out-of-pocket payments are absent in our case study, the insurance dummy variable does not capture the effect of a lessened individual budget constraint. As far as its role is to control for the preference structure of the woman, the sign of its coefficient is not predictable in both equations. ${ }^{8}$

Theoretical identification of the recursive probit model is achieved as soon as both equations of the model contains a varying exogenous regressor (Wilde, 2000). However, to avoid that identification strongly relies on model's functional form we use as instruments a set of dummy variables conveying information on the type of residential area, ranging from central metropolitan to small rural area. As hospital choice is influenced by the local availability of providers, these indicators are meant to control for discrepancies in the choice set of hospitals considered by the woman. We also include as instrument in the hospital choice equation a dummy indicating whether the woman has a self-employed occupation. As a robustness check, we estimated the model without any exclusion restriction and found very similar results, showing that conditionally on the other explanatory variables, our extra regressors do not influence the probability of CS scheduling.

Given the self-compiled nature of the questionnaire our set of risk factors do not include most of the clinical conditions usually controlled for in the health econometrics analysis of CS variation (see for

\footnotetext{
${ }^{8}$ It might be argued that the insurance dummy is potentially endogenous in the treatment equation, as suggested by Mossialos et al. (2005). Accounting for endogeneity of insurance would require a more general model with a third equation explaining insurance ownership. Given the difficulties in performing reliable inference on exogeneity in small samples even in the bivariate context (Monfardini and Radice, 2006), we consider our sample not appropriate for this exercise and leave this issue for future research.
} 
example Dubay et al., 1999). Major lacks are controls for breech presentation, fetal distress and prior CS. The latter variable is known to be a major predisposing factor for CS delivery. In order to overcome this limitation we exploit information about primiparity. However we are only able to approximately identify primiparae women. We code as primipara a woman with no other natural children living in their family older than that the surveyed delivery refers to. This strategy is quite plausible provided that in Italy almost all children are placed in the care of their mother in case of parents divorce. According to this identification criterion, primiparas are about $40 \%$ of our national sample, a "realistic" proportion in Italy. We include this dummy for primiparity and its interaction with the dummy indicating whether the woman is aged more than 36 in a second specification of the model. We report the estimation results for both specification as Model 1 and Model 2 hereafter.

\section{INSERT TABLE 2 HERE}

\subsection{Main results}

Table 3 presents the main findings emerging from the following specifications: univariate probit, seemingly unrelated bivariate probit, and recursive probit model. To obtain MLE of the latter models, we resorted to the command "biprobit" of STATA 9, which exploits the Newton-Raphson maximization method and allows for Hessian-based estimation of the asymptotic covariance matrix. Such command, presented in STATA only for the SURE bivariate probit, sorts out the correct estimation procedure also when one of the dependent dichotomous variable is included as a regressor for the other probit equation, as the two models share the same log-likelihood "mechanics". In the recursive probit model the PRIVATE dummy proves to be positive and highly significant, picking up hospital specific factors that increases the probability of a scheduled CS. To evaluate the exogeneity status of this dummy we compute alternative exogeneity tests analysed in Monfardini and Radice (2006): conditional moments (CM), different versions of the lagrange multiplier test (LM1, LM2, LM3, LM4), likelihood ratio (LR) and the Wald-type test based on the esimated value of the correlation coefficient (RHO). As expected, we find that the dummy is endogenously codetermined with the scheduled CS equation. The battery 
of exogeneity tests presented in the bottom part of the table provides conflicting indications at a first sight. The CM, LM1, LR and RHO tests lead to strong rejection of the hypothesis of exogeneity, while LM2, LM3 and LM4 support the opposite evidence, i.e. in favour of exogeneity of the hospital type dummy. However, the Monte Carlo evidence presented in Monfardini and Radice (2006), helps in distinguishing and interpreting these results, as the latter set of tests exhibit finite sample distributions remarkably far from the asymptotic ones. This leads us to conclude that in our case study the bivariate endogenous dummy model is the appropriate setting for drawing some consistent inference on hospital type differences in CS scheduling rates.

\section{INSERT TABLE 3 HERE}

A full account of the bivariate endogenous dummy probit estimation exercise is available in the Appendix. For the sake of brevity we only notice here that overall results are coherent with expected signs. Each risk factor contributes to increase the probability of scheduling a CS, while they are almost uniformly not significant in driving hospital choice. A noticeable exception is represented by newborn weight: babies with low weight at birth are less frequently delivered in a private hospital. Socioeconomic variables (education) seem to be irrelevant in determining CS planning probability with the exception of insurance holding. However, being self-employed, holding a private health insurance and being more educated makes the woman to have a higher probability to deliver in a private hospital. The coefficients of the primipara dummies in Model 2 imply that a woman delivering for the first time is less likely to deliver with a scheduled CS when younger than 36, but more likely to do so when aged more than 36 . In broader terms, if we restrain ourselves to the individual observable effects, it seems that scheduling is driven, as expected, by some relevant risk factor but is less so by socio-economic variables. The reverse applies to the decision to opt for a private admission. Even more coarsely we could say that according to observables CS scheduling is a clinical matter and opting to a private hospital has to do with socio-economics.

Turning to the two main coefficients of interest, the following comments apply. The negative and significant correlation coefficient suggests that among the two self-selection forces we figured out in 
section 3 , the one related to preferences is dominated by the unobserved frailty one. This allocates the more risky patients to public hospitals, i.e. the higher quality hospitals. The significant and positive dummy coefficient indicates that, net of observable and unobservable confoundings, we measure a significantly higher inclination to practice CS scheduling in private hospitals. This is, as we suggested, a precondition to interpret the correlation coefficient in the light of assortative mating mechanism. Because of its measured negative sign, we conclude that assortative mating is of minor relevance in our case, even if we cannot exclude it to be present. It is worth noticing that according to the bivariate SURE probit model the estimated correlation is positive and therefore apparently coherent with an opposite interpretation of the self-selection process at work. As the SURE model is actually nested in the endogenous dummy one, we are able to conclude that the former is rejected, with the coefficient of the dummy being significantly different from zero. ${ }^{9}$

\section{INSERT TABLE 4 HERE}

In Table 4 we look at the appropriate scheduled CS probability differentials to evaluate the impact of the PRIVATE hospital dummy for a set of representative women. These are characterized by different risk factors and primiparity status. Given the lack of major risk factors in our specification, the considered profiles describe intermediate levels of riskiness $r_{i}$. Therefore, in line with our intepretative model of section 2 , these representative women may concretely switch to a private hospital. Incidentally we notice that, coherently with our framework of section 2 , the more risky woman is less likely to refer to a private hospital. For all typical women the impact of the PRIVATE hospital dummy is positive and significant as emerges from column (6) where we evaluate the standard error of the difference through the Delta method. Our low risk primipara has a probability of $8.94 \%$ of getting a scheduled CS in public hospitals, increasing to $15.82 \%$ when she refers to a private one. ${ }^{10}$ Our assertion on public hospitals practice as representing the appropriate, professional norm suggests to look for a measure useful for

\footnotetext{
${ }^{9}$ The endogenous dummy variable model is therefore the appropriate one to conduct a meaningful profiling analysis of healthcare providers (see Normand et al., 1997) acknowledging the public/private difference.

${ }^{10}$ It is worth noticing here that these figures lie below the target set for low risk primiparas in the US plan "Tracking Healthy People 2010" (see US Department of Health and Human Resources, 2000).
} 
evaluating the extent to which private hospitals move away from this golden standard. To this purpose, we compute the percentage change of the probability of receiving a scheduled CS in private with respect to the corresponding figure in public hospitals. For the primipara high risk woman such percentage difference is equal to $42 \%$. For her low risk counterpart the percent change reaches a huge $77 \%$, i.e. almost doubled. The observed percentage difference across risk profiles is enormous indicating that in our case study private hospitals scheduling practice exceeds the public norm the more the less risky is the patient.

\section{Conclusions}

We study practice variation in scheduling of cesarean section delivery across public and private hospitals. In the health economics literature the prevailing approach ascribes variation in CS adoption to physician unilateral response to a broad set of economic incentives. We adopt here a novel perspective and look at the role played by patients' preferences for the treatment, allowing for the presence of an assortative mating process driven by provider style of practice. We discuss which circumstances make it feasible an empirical assessment of assortative mating in healthcare markets and argue that our case study is well suited to this purpose.

The econometric model adopted for the endogenous discrete variable represented by treatment is Heckman's recursive probit model. The analysis of practice variation across alternative providers is performed by including among the determinants for the probability of the treatment, a dummy variable indicating the provider chosen by the patient. The latter is determined by an individual choice process in which patients' preferences for the alternative treatments and information on provider's style of practice play a major role. This brings about self-selection of patients into providers based on observables and unobservables characteristics that also determine the given treatment, and makes the provider dummy variable potentially endogenous. Unobserved variables are both related to patient's preferences and unobserved severity conditions. The first set originates a nonrandom selection which has to do with assortative mating, and implies a positive correlation coefficient. However, this effect can be partially 
or totally offset by a self-selection of opposite sign introduced by the second set of unobservables. The negative sign of the latter is a mantained assumption that finds sound justification in the higher quality of public hospitals, attracting women with more severe unobservable conditions.

In our case study on an Italian sample we obtain strong evidence against the hypothesis of exogeneity of hospital type dummy in the equation determining CS scheduling probability. Our results suggest that a self-selection mechanism allocating the more risky patients to public hospitals is prevailing over the assortative mating mechanism operating through unobservable preferences for the treatment. After controlling for observable and unobservable characteristics, women admitted to a private hospital are more likely to receive a scheduled CS at any risk profile. Thus, working in a private hospital seems to insulate the physicians from the adherence to a prevailing professional norm set by their public hospital counterparts. Looking at the percentage change of the probability of receiving a scheduled CS in private with respect to the corresponding figure in public hospitals, we find that in our case study private hospitals scheduling practice exceeds the public norm the more the less risky is the patient. 


\section{References}

[1] Agency for Healthcare Research and Quality. 2002. AHRQ quality indicators - Guide to inpatient quality indicators: quality of care in hospitals - volume, mortality, and utilization. Rockville, MD. Revision 2.

[2] Al-Mufti R, McCarthy A, Fisk NM. 1997. Survey of obstetricians' personal preference for discretionary practice. European Journal of Obstetrics and Gynecology, 73, 1-4.

[3] Benitez-Silva H, Buchinsky M, Chan H-M, Rust J, and Sheidvasser S. 2004. How Large is the Bias in Self-Reported Disability Status? Journal of Applied Econometrics, 19(6), 649-70.

[4] Brooks JM, Dor A and Wong HS. 1997. Hospital-insurer bargaining: an empirical investigation of appendectomy pricing. Journal of Health Economics, 16, 417-34.

[5] Buchmueller TC, Couffinhal A, Grignon M, Perronin M. 2004. Access to physician services: does supplemental insurance matter? Evidence from France. Health Economics, 13, 669-87.

[6] Cromwell J, Mitchell J. 1986. Physician-induced demand for surgery. Journal of Health Economics, 5, 293-313.

[7] Dubay L, Kaestner R, Waidmann T. 1999. The impact of malpractice fear on cesarean section rates. Journal of Health Economics, 18, 491-522.

[8] Epstein, A, Nicholson, S. 2005. The formation and evolution of physician treatment styles: an application to cesarean sections. NBER Working Paper $\mathbf{1 1 5 4 9 .}$

[9] Frank RG. 2004. Behavioral Economics and Health Economics. NBER Working Paper 10881.

[10] Geweke J, Gowrisankaran G, Town RJ. 2003. Bayesian Inference for Hospital Quality in a Selection Model. Econometrica, 71, 1215-38.

[11] Gruber J, Kim J, Mayzlin D. 1999. Physician fees and procedure intensity: the case of cesarean delivery. Journal of Health Economics, 18, 473-90. 
[12] Gruber J, Owings M. 1996. Physician financial incentives and cesarean section. Rand Journal of Economics, 27, 99-123.

[13] Heckman J. 1978. Dummy endogenous variables in a simultaneous equation system. Econometrica, 46, 931-59.

[14] Holly A, Gardiol L, Domenighetti G, Bisig B. 1998. An econometric model of health care utilization and health insurance in Switzerland. European Economic Review, 42, 513-522.

[15] Lo JC. 2003. Patients' attitudes vs. physicians' determination: implications for cesarean sections. Social Science \& Medicine, 57, 91-6.

[16] MacKenzie IZ. 1999. Should women who elect to have cesarean sections pay for them? British Medical Journal, 318, 1070.

[17] Maddala GS. 1983. Limited Dependent and Qualitative Variables in Econometrics.Cambridge University Press: Cambridge.

[18] Maude-Griffin R, Feldman R and Wholey D. 2004. Nash bargaining model of HMO premiums. Applied Economics, 36, 1329-36.

[19] McGuire TG, Pauly MV. 1991. Physician response to fee changes with multiple payers. Journal of Health Economics, 10, 385-410.

[20] Minkoff, H and Chevernak, FA, 2003. Elective primary cesarean delivery. The New England Journal of Medicine, 348, 946-950.

[21] Monfardini, C. and Radice, R. 2006. Testing exogeneity in the bivariate probit model: a Monte Carlo study. Available at SSRN: http://ssrn.com/abstract=886506

[22] Mossialos E, Costa-Font J, Davaki K and Karras K. 2005. Is there 'patient selection' in the demand for private maternity care in Greece? Applied Economics Letters, 12, 7-12.

[23] Normand S-LT, Glickman ME, Gatsonis CA, 1997. Statistical methods for profiling providers of medical care: issues and applications. Journal of the American Statistical Association, 92, 803-14. 
[24] Paterson-Brown S. 1998. Should doctors perform an elective caesarean section on reqest? Yes as long as the woman is fully informed. British Medical Journal, 317, 462-5.

[25] Phelps CE, Mooney C. 1993. Variations in medical practice use: causes and consequences. In: Arnould RJ, Rich RF, White W (eds.), Competitive approaches to health care reform. The Urban Institute Press, Washington, DC. 139-78.

[26] Phelps, CE. 2000. Information diffusion and best practice adoption. In: Cuyler, AJ, Newhouse, JP, eds., Handbook of Health Economics, Vol. 1A. Amsterdam: Elsevier Science, 223-64.

[27] Ryan M, Hughes J. 1997. Using conjoint analysis to assess women's preferences for miscarriage management. Health Economics, 6, 261-73.

[28] San Miguel F, Ryan M, McIntosh E. 2000. Applying conjoint analysis in economic evaluations: an application to menorrhagia. Applied Economics, 32, 823-33.

[29] US Department of Health and Human Resources. 2000. Tracking Healthy People 2010. Washington DC.

[30] Wennberg JE, Gittelsohn A. 1973. Small area variations in health care delivery. Science, 182, 1102-08.

[31] Wilde J. 2000. Identification of multiple equation probit models with endogenous dummy regressors. Economics Letters, 69, 309-12.

[32] World Health Organization. 1985. Recommendation on appropriate technology for Birth. Document approved in the WHO Conference at Fortaleza, Brazil, 22-26 april 1985. 


\section{TABLES TO BE INSERTED IN THE MAIN TEXT}

\section{Table 1. Cesarean section (CS) incidence across hospital types}

\begin{tabular}{lccccc}
\hline \hline & CS rate & $\begin{array}{c}\text { Scheduled CS } \\
\text { rate }\end{array}$ & $\begin{array}{c}\text { CS rate } \\
\text { conditional on } \\
\text { laboring }\end{array}$ & & $\begin{array}{c}\text { "Market" } \\
\text { Shares }\end{array}$ \\
\cline { 2 - 4 } \cline { 5 - 6 } Public hospital & $27.5 \%$ & $16.9 \%$ & $12.8 \%$ & & $91.4 \%$ \\
Private hospital & $42.4 \%$ & $32.3 \%$ & $14.9 \%$ & & $8.6 \%$ \\
All & $28.8 \%$ & $18.2 . \%$ & $12.9 \%$ & & $100.0 \%$ \\
\hline \hline
\end{tabular}

\section{Table 2. Variables description}

\begin{tabular}{|c|c|}
\hline Variable & \\
\hline Scheduled & $=1$ if woman delivers with a scheduled cesarean section; $=0$ otherwise \\
\hline Private & $=1$ if woman delivers in a private hospital; $=0$ otherwise \\
\hline \multirow{2}{*}{ Primipar } & Risk Factors \\
\hline & $=1$ if woman delivers for the first time; $=0$ otherwise \\
\hline Amniocen & $\begin{array}{l}=1 \text { if the woman underwent early prenatal diagnostic checks ("villi coriali" or "amniocentesi"); }=0 \\
\text { otherwise }\end{array}$ \\
\hline Diabetes & $=1$ if the woman self-reports having suffered from diabetes during her pregnancy; $=0$ otherwise \\
\hline Gestosis & $=1$ if the woman self-reports having suffered from "gestosi" during her pregnancy; $=0$ otherwise \\
\hline Hyperten & $\begin{array}{l}=1 \text { if the woman self-reports having suffered from blood hypertension during her pregnancy; }=0 \\
\text { otherwise }\end{array}$ \\
\hline BMI & Body Mass Index (=bodyweight/(height/100)²) \\
\hline Newborn weigth & weight of the newborn in kilograms \\
\hline Newborn weigth sq & weight of the newborn squared \\
\hline No. scans & number of fetal ultrasound scans done during pregnancy \\
\hline Smoked & $=1$ if the woman was an abitual smoker; $=0$ otherwise \\
\hline Age +36 & $=1$ if woman is older than $36 ;=0$ otherwise \\
\hline Age & age in years \\
\hline \multirow[t]{2}{*}{ Agesq } & age squared \\
\hline & Socio-economic variables \\
\hline Edu-high & $=1$ if woman holds an high education degree; $=0$ otherwise \\
\hline Edu-low & $=1$ if woman holds a low education degree; $=0$ otherwise \\
\hline Edu-medium & $=1$ if woman holds a medium education degree; $=0$ otherwise \\
\hline Insured & $=1$ if the woman is covered by private health insurance \\
\hline \multirow[t]{2}{*}{ Self-employed } & $=1$ if the woman is self-employed; $=0$ otherwise \\
\hline & Other controls \\
\hline NW & $=1$ if the woman resides in a North-West region; $=0$ otherwise \\
\hline NE & $=1$ if the woman resides in a North-East region; $=0$ otherwise \\
\hline CEN & $=1$ if the woman resides in a Centre region; $=0$ otherwise \\
\hline ISL & $=1$ if the woman resides in a Island region (Sicily or Sardinia); $=0$ otherwise \\
\hline Area-metropol & $=1$ if the woman resides in a metropolitan area; $=0$ otherwise \\
\hline Area-suburban & $=1$ if the woman resides in a metropolitan suburb; $=0$ otherwise \\
\hline Area-small & $=1$ if the woman resides in a very small commune (less than 2000 inhabitants); $=0$ otherwise \\
\hline Area-medium & $\begin{array}{l}=1 \text { if the woman resides in a medium-small commune (between } 2000 \text { and } 10000 \text { inhabitants); =0 } \\
\text { otherwise }\end{array}$ \\
\hline
\end{tabular}


Table 3. Main results

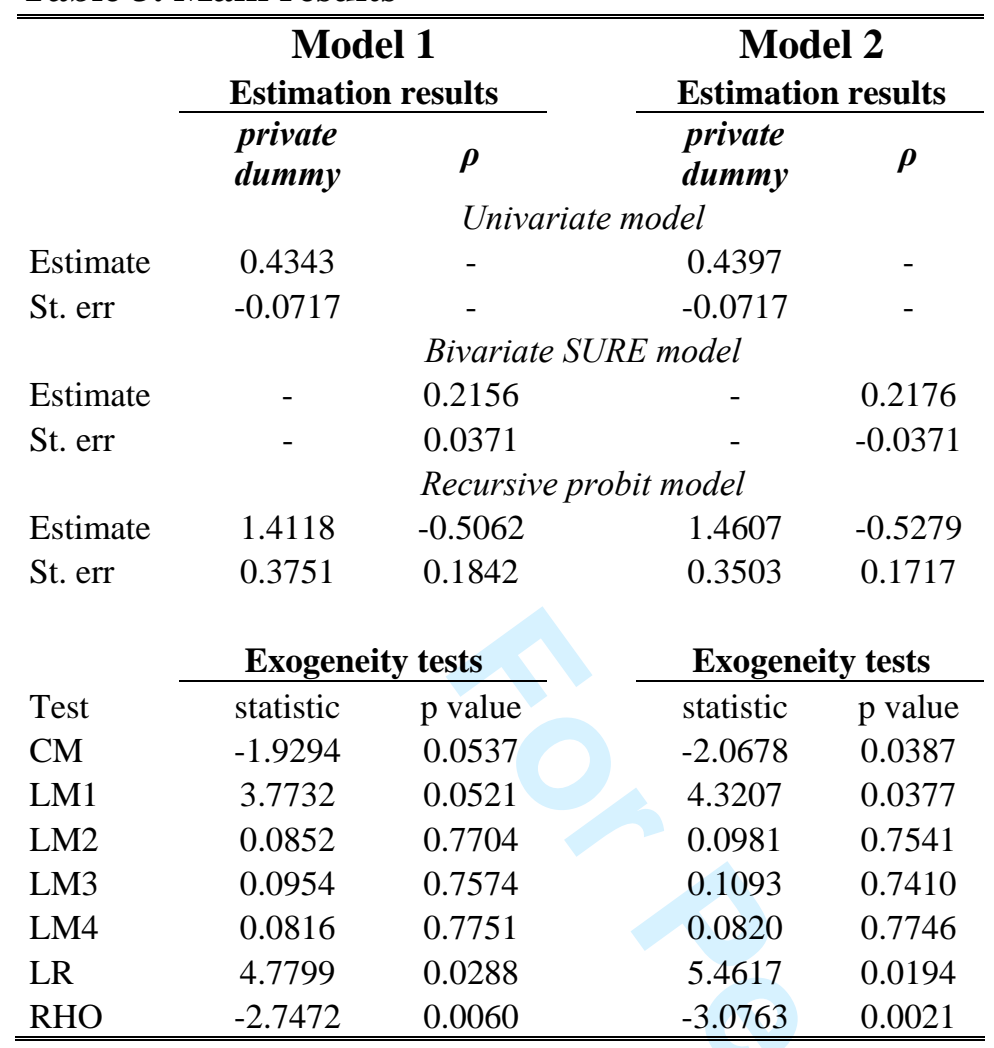


Table 4

Predicted effect of hospital type dummy on probability of scheduling CS

\begin{tabular}{|c|c|c|c|c|c|}
\hline $\begin{array}{c}(1) \\
\text { Woman type }\end{array}$ & $\begin{array}{c}\text { (2) } \\
\text { Pr(priv) }\end{array}$ & $\begin{array}{c}(3) \\
\operatorname{Pr}(C S)\end{array}$ & $\begin{array}{c}\text { (4) } \\
\operatorname{Pr}(C S \mid \text { priv) }\end{array}$ & $\begin{array}{c}\text { (5) } \\
\operatorname{Pr}(\text { CS } \mid \text { pub) }\end{array}$ & $\begin{array}{c}\text { (6) } \\
\text { Difference (s.e.) }\end{array}$ \\
\hline & \multicolumn{5}{|c|}{ Irrespective of primiparity (based on Model 1) } \\
\hline Low risk & 0.0958 & 0.1100 & 0.1811 & 0.1025 & $0.0786 * * *(0.0262)$ \\
\hline \multirow[t]{2}{*}{ High risk } & 0.0585 & 0.3270 & 0.4495 & 0.3193 & $0.1302 * * \quad(0.0499)$ \\
\hline & \multicolumn{5}{|c|}{ Primipara (based on Model 2) } \\
\hline Low risk & 0.0946 & 0.0959 & 0.1582 & 0.0894 & $0.0688 * * \quad(0.0262)$ \\
\hline \multirow[t]{2}{*}{ High risk } & 0.0573 & 0.2997 & 0.4153 & 0.2927 & $0.1226 * * \quad(0.0520)$ \\
\hline & \multicolumn{5}{|c|}{ Multipara (based on Model 2) } \\
\hline Low risk & 0.0966 & 0.1311 & 0.2137 & 0.1222 & $0.0915 * * *(0.0289)$ \\
\hline High risk & 0.0587 & 0.3660 & 0.4978 & 0.3578 & $0.1400 * * *(0.0515)$ \\
\hline
\end{tabular}

(1)

Low risk woman is characterized by the absence of clinical risk (all the dummy variables indicating severity of the pregnancy set to zero); variables age, No. scans, BMI, newborn weight set to sample averages; medium education degree; without private insurance, not self-employed, delivering in 1996, residing in the North-East of Italy in a metropolitan area

High risk woman differs from the previous for the following risk factors: newborn weight equal to $2.5 \mathrm{Kg}$, BMI=30, suffers from gestosis

(2)

Marginal probability of referring to private hospital, conditional to the explanatory variables $x$. Conditioning to $x$ is omitted from notation in all column headings.

(3)

Marginal probability of delivering with scheduled CS, conditional to the explanatory variables $x$.

(4)

Probability of planning c-section conditional to referring to private hospital (and to explanatory variables $x$ ), evaluated as: $\operatorname{pr}\left(p l_{-} C S=1 \mid \operatorname{priv}=1\right)=\operatorname{pr}\left(p l_{-} C S=1, \operatorname{priv}=1\right) / \operatorname{pr}(\operatorname{priv}=1)$ through the appropriate bivariate and univariate normal cumulative distribution function.

(5)

Probability of planning c-section conditional to referring to public hospital (and to explanatory variables $x$ ), evaluated as: $\operatorname{pr}\left(p l_{-} C S=1 \mid \operatorname{priv}=0\right)=p r\left(p l_{-} C S=1, \operatorname{priv}=0\right) / \operatorname{pr}(\operatorname{priv}=0)$

(6)

The variance of the estimated difference between the two conditional probabilities has been evaluated through the Delta Method, exploiting analytical expressions of first order derivatives of the bivariate and univariate normal cumulative distribution function. The details of computation are available upon request. 


\section{APPENDIX}

Table A.1

Descriptive statistics

\begin{tabular}{|c|c|c|c|c|c|c|}
\hline \multirow[b]{2}{*}{ Variable } & \multicolumn{2}{|c|}{ Full sample } & \multicolumn{2}{|c|}{$\begin{array}{c}\text { Public hospital } \\
\text { admissions (PRIV==0) }\end{array}$} & \multicolumn{2}{|c|}{$\begin{array}{c}\text { Private hospital } \\
\text { admissions (PRIV==1) }\end{array}$} \\
\hline & Mean & Std. Dev. & Mean & Std. Dev. & Mean & Std. Dev. \\
\hline Private & 0.086 & 0.279 & 0.000 & & 1.000 & \\
\hline Primipar & 0.471 & 0.499 & 0.471 & 0.499 & 0.473 & 0.499 \\
\hline Diabetes & 0.019 & 0.137 & 0.019 & 0.135 & 0.023 & 0.151 \\
\hline Gestosis & 0.037 & 0.189 & 0.039 & 0.193 & 0.021 & 0.142 \\
\hline Hyperten & 0.046 & 0.209 & 0.048 & 0.214 & 0.026 & 0.159 \\
\hline BMI & 22.69 & 3.455 & 22.72 & 3.475 & 22.52 & 3.238 \\
\hline Newborn weigth & 3.263 & 0.509 & 3.265 & 0.515 & 3.239 & 0.437 \\
\hline No. scans & 5.378 & 2.319 & 5.359 & 2.322 & 5.579 & 2.275 \\
\hline Amniocen & 0.238 & 0.426 & 0.232 & 0.422 & 0.305 & 0.461 \\
\hline Smoked & 0.240 & 0.427 & 0.239 & 0.427 & 0.248 & 0.432 \\
\hline Age & 32.17 & 4.988 & 32.170 & 4.943 & 32.21 & 5.452 \\
\hline Age +36 & 0.249 & 0.432 & 0.244 & 0.429 & 0.305 & 0.461 \\
\hline Edu-high & 0.108 & 0.311 & 0.103 & 0.304 & 0.168 & 0.374 \\
\hline Edu-medium & 0.467 & 0.499 & 0.464 & 0.499 & 0.499 & 0.501 \\
\hline Edu-low & 0.367 & 0.482 & 0.373 & 0.484 & 0.299 & 0.459 \\
\hline Insured & 0.157 & 0.364 & 0.155 & 0.362 & 0.176 & 0.381 \\
\hline Self-employed & 0.441 & 0.497 & 0.432 & 0.495 & 0.537 & 0.499 \\
\hline NW & 0.176 & 0.381 & 0.185 & 0.388 & 0.085 & 0.279 \\
\hline $\mathrm{NE}$ & 0.213 & 0.409 & 0.222 & 0.416 & 0.119 & 0.324 \\
\hline CEN & 0.158 & 0.365 & 0.161 & 0.367 & 0.124 & 0.330 \\
\hline ISL & 0.129 & 0.335 & 0.126 & 0.332 & 0.129 & 0.336 \\
\hline Area-metropol & 0.081 & 0.273 & 0.074 & 0.262 & 0.155 & 0.362 \\
\hline Area-suburban & 0.096 & 0.295 & 0.093 & 0.291 & 0.127 & 0.333 \\
\hline Area-small & 0.186 & 0.389 & 0.196 & 0.397 & 0.080 & 0.272 \\
\hline Area-medium & 0.286 & 0.452 & 0.288 & 0.453 & 0.266 & 0.443 \\
\hline
\end{tabular}


Table A.2

Full estimation results

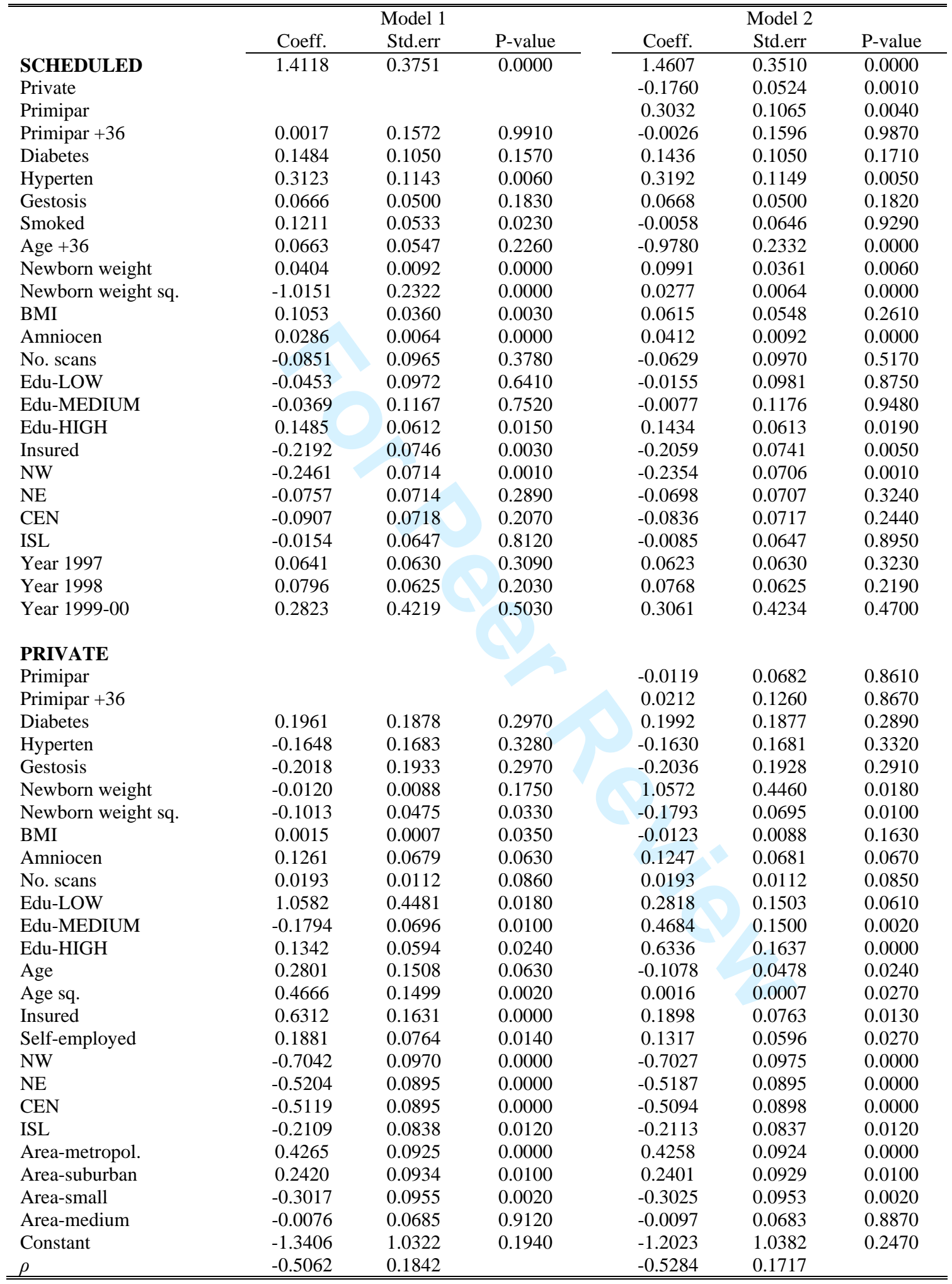

